REVISTA 89.1

Revista RELACIONES INTERNACIONALES

Escuela de Relaciones Internacionales.

Universidad Nacional, Costa Rica.

N. ${ }^{\circ} 89.1$ • Enero-Junio de 2016

doi: http://dx.doi.org/10.15359/ri.89-1.4

Pp. 77-93

\title{
LA ALIANZA DEL PACÍFICO: ¿HACIA EL CAMINO DE LA INTEGRACIÓN?
}

\author{
Víctor Alejandro Godoy López*
}

\section{RESUMEN}

Los países de América Latina, históricamente, no han contado con relaciones fuertes con el continente asiático como consecuencia del direccionamiento histórico de sus relaciones internacionales hacia el hemisferio occidental Desde que los países de la región lograron su independencia de España, estuvieron, en primera medida, influenciados por Inglaterra y luego por Estados Unidos. Precisamente, salvo algunas excepciones, como Chile o Perú, fue en los primeros años de la década de 1990 que América Latina y Asia Pacífico empezaron a mejorar sus vínculos comerciales. La región comenzó a ver a Asia como un posible modelo económico a seguir y, también, como una alternativa para la diversificación de su política exterior.

Palabras clave: Alianza del Pacífico, América Latina, integración, desarrollo, retos.

\begin{abstract}
:
Historically, Latin American countries have not had strong relations with Asia as a consequence of their international relations directing towards the Western hemisphere; this is a result of Latin American countries, upon gaining independence from Spain, having been influenced first by England and then by the United States. Indeed, save a few exceptions such as Chile and Peru, it was only in the early 1990s that Latin America and Asia Pacific began to improve trade relations. The Latin American region began to see Asia as a possible economic model and an alternative for the diversification of its foreign policy.

Keywords: The Pacific Alliance; Latin America; Integration; Development Challenges.
\end{abstract}

* Comunicador Social y Periodista, Magister en Logística Integral y Comercio Exterior, Universidad Camilo José Cela; Magíster en International Affaires, Universidad Externado de Colombia y Columbia University. Docente Universidad Santo Tomás Sede Bogotá. Correo electrónico: victorgodoy@ usantotomas.edu.co 


\section{Introducción}

El creciente interés de América Latina por fortalecer las relaciones con Asia se deriva, precisamente, de la necesidad de diversificar su comercio, de entrar en contacto con nuevas economías y empezar a encontrar espacios de mayor visibilidad en el ámbito comercial, que generen réditos económicos, en primera instancia, y luego, con el tiempo, una mayor visibilidad a nivel político, en las relaciones con Europa, África y Asia.

Precisamente América Latina ha mirado poco más allá de China o Japón para sus relaciones comerciales. A nivel histórico, sin embargo, no han sido relaciones fluidas; pero, en los últimos años, América Latina ha empezado a verlos como unos socios importantes que le sirven para sus relaciones con el nordeste de Asia (Velosa, 2014).

Durante la Guerra Fría, América Latina vivió un aislamiento, debido a la presencia única de Estados Unidos en la búsqueda por impedir que los países de la región se acercaran ideológicamente a China o a la Unión Soviética en el marco de su tendencia política socialista. Para que América Latina empezara a desmarcarse de la política de Estados Unidos, se han establecido las siguientes situaciones (Fernández de Soto \& Pineda, 2012):

La importancia de Estados Unidos para América Latina disminuyó, debido al surgimiento de opciones políticas que quieren establecer un cambio en el manejo de las relaciones entre ambas regiones, ejemplo de esta situación es el llamado "socialismo del siglo XXI", donde el expresidente venezolano Hugo Chávez, llamó a un cambio de la dependencia con Estados Unidos.

Por consiguiente, en la actualidad no está definido el tipo de integración a la que la región le apuesta, sí un área de libre comercio, o un área delimitada como la Unión de Naciones Suramericanas (UNASUR) con Estados Unidos o sin este país. Tal es el caso de la Alianza Bolivariana para los Pueblos de Nuestra América (ALBA), o la integración según los tratados de libre comercio o los antiguos bloques como la Comunidad Andina de Naciones (CAN), o el Mercado de Naciones Suramericanas (MERCOSUR). En consecuencia, la crisis de integración demuestra que en América Latina no existe una voz uniforme sobre las estrategias ni los fines que se buscan a futuro, quizás por los múltiples conceptos de integración que se manejan hoy.

Por otra parte, la visibilidad de las economías emergentes es cada vez mayor, destacándose el papel de Brasil, Rusia, India, China y Suráfrica que conforman los (BRICS); ya para el año 2009, estos países suman el $95.5 \%$ 
del PIB norteamericano, y reúnen 2.700 millones habitantes, lo que representa más del $40 \%$ de la población mundial (Corvalán, 2011).

De esta forma, las actividades comerciales y de inversión entre América Latina y Asia-Pacífico continúan expandiéndose, gracias, sobre todo, al gran incremento de las corrientes comerciales. Los vínculos económicos birregionales en general siguen siendo débiles o reflejan una escasa diversificación del comercio. De esta manera, el Foro de Cooperación Económica Asia-Pacífico, conocido como (APEC) por sus siglas en inglés, Consejo Económico de la Cuenca del Pacífico, conocida por sus siglas en inglés como (PBEC) y la Asociación de Naciones del Sudeste Asiático (ASEAN), por sus siglas en inglés, se han convertido en los principales grupos de cooperación, y destacan los esfuerzos de coordinación de la región, con la intención de proyectarse hacia el Asia-Pacífico más integrada.

Para la mayoría de los países de América Latina, Asia-Pacífico es un mercado sin explotar, a pesar de su impresionante desempeño en áreas como el crecimiento, el comercio internacional, la inversión extranjera directa; modernización tecnológica y capacidad de innovar; y las crecientes reservas internacionales que posee. La dinámica actual de la demanda agregada de los países de Asia-Pacífico, principalmente China, ofrece oportunidades sin precedentes a la región de América Latina y el Caribe en el ámbito productivo y exportador, tanto de productos básicos como de manufacturas y servicios (Fernández de Soto \& Pineda, 2012).

Así como se ha dado un modelo de acrónimos que enlaza los nuevos mercados emergentes, el peso de los bloques regionales de integración y cooperación se ha incrementado. Sin embargo, es complicado rastrear las causas del fracaso de la integración regional, ya que el panorama estratégico no lo establece como un único proceso ni con un camino lineal. En los 50 años transcurridos desde el inicio de la integración latinoamericana, ha surgido un gran número de instituciones, cada una de ellas con sus siglas correspondientes. De hecho, la integración se ha convertido en un cúmulo de subprocesos, regionales y subregionales, que incluso se contradicen entre sí (Malamud, 2009).

Fue en el año 2006 cuando surge la propuesta de promover la integración y cooperación entre los países del Pacífico en América Latina (integrada por 11 países, a saber: Chile, Colombia, Costa Rica, Ecuador, El Salvador, Guatemala, Honduras, México, Nicaragua, Panamá y Perú, dándole pauta a la Alianza del Pacífico). Dicha iniciativa comienza a discutirse por el Gobierno Peruano cuando se suscita la crisis que generó en la CAN (Comunidad Andina de Naciones) la decisión del Presidente Hugo Chávez de retirar a Venezuela de este bloque. 
Así surge el Arco del Pacífico en un intento de asegurar una posición regional frente al grupo dinámico de economías de la Cuenca del Pacífico, cuya influencia en América Latina es cada vez mayor (Malamud, Infolatam, 2015).

Por consiguiente, seis años después, en junio de 2012 se constituyó oficialmente la Alianza del Pacífico, con un enfoque de libre mercado a la integración regional, y ampliamente percibida como una alternativa que en el futuro pueda generar un diálogo de gobernabilidad, cooperación e integración en la región.

A nivel interno, en Colombia, el gobierno del presidente Juan Manuel Santos, con la idea de mostrar al mundo un país que se encuentra inmerso en la globalización, profundiza la inserción de flujos económicos, comerciales y políticos del Asia y Pacífico. China, Rusia, Turquía e India son países que, en los próximos años, se busca y espera cumplan un papel como nuevos aliados. De igual manera, se fortalecerá el diálogo multilateral y bilateral con los países de la región, aumentando las visitas recíprocas de alto nivel en todas las áreas. La agenda se concentrará en negociaciones comerciales y de inversión con la comunidad de países del Golfo Pérsico, Japón, Turquía, Rusia y Corea del Sur. Además de la inserción económica, un escenario ideal es el Foro de Cooperación América Latina - Asia del Este (FOCALAE) para trabajar temas políticos, educativos, sociales, culturales, tecnológicos y de turismo.

\section{Recorrido histórico de la integración latinoamericana}

Con un interés de mayor unidad en las negociaciones, desde mediados de los años 60, América Latina busca establecer un modelo de integración donde las políticas de intercambio comercial sean armónicas y permitan, a los países que se han asociado en bloques, un crecimiento de manera conjunta, en consecuencia, se estable en primera instancia la Asociación Latinoamericana de Libre Comercio, ALALC, el 18 de febrero de 1960, como propuesta de integración económica latinoamericana, con la participación de Brasil, Argentina, Chile, México, Paraguay, Uruguay y Perú. Luego se vinculan Bolivia, Colombia, Ecuador y Venezuela. Este proceso de integración tiene como objetivo crear una zona de libre comercio, que, en principio, debe estar en funcionamiento en un periodo de (12) doce años, lo que se prolongó a (20) veinte y se convirtió, en 1980, en la Asociación Latinoamericana de Integración (ALADI) (Banco Interamericano de Desarrollo, 2014).

A partir de 1965, como estructura institucional de integración en América Latina, surge el Instituto para la Integración de América Latina y el Caribe 
(INTAL/BID) (Tirado, 1997). Su objetivo principal es generar y difundir conocimiento sobre las ventajas de los procesos de integración. A partir del año 1975 se creó el Sistema Económico Latinoamericano y del Caribe (SELA) (Banco Interamericano de Desarrollo, 2011), que promueve un sistema de consulta y coordinación para concertar posiciones y estrategias comunes, en materia económica, ante países, grupos de naciones, foros y organismos internacionales e impulsa la cooperación y la integración entre países de América Latina y el Caribe.

Con el fin de proseguir con la integración de la región, en 1969 se establece el Acuerdo de Cartagena-Pacto Andino, que agrupó en un comienzo a Bolivia, Colombia, Chile, Ecuador y Perú, pero más tarde ingresó Venezuela en 1973 y se retiró Chile en 1976, debido a la llegada de la dictadura de Augusto Pinochet y un modelo de política ligado al naciente esquema neoliberal. Sin embargo, las dificultades políticas de los años 70 y 80 , y el proceso autoritario en Chile, Argentina, Uruguay, Paraguay, entre otros, debilitaron este proceso que, finalmente, recibió el nombre de Comunidad Andina de Naciones, en junio de 1997. Ha tenido, como objetivos fundamentales, profundizar la integración subregional andina, promover su proyección externa y robustecer las acciones relacionadas con el proceso de integración. La Zona de Libre Comercio Andina se inició en 1969 y culminó en 1993. Para ello aplican como mecanismo principal el Programa de Liberación, encaminado a eliminar todos los gravámenes aduaneros e impuestos que inciden sobre las importaciones. No obstante, una de las grandes críticas realizadas sobre este tema, es la imposibilidad de que más allá del papel, se logre este objetivo central en este experimento de la integración (Comunidad Andina de Naciones, 2014).

Luego, se presentó la posibilidad de establecer la Asociación Latinoamericana de Integración (ALADI), (Asociación Latinoamericana de Integración, Acuerdo \# 1, 2014), como un Tratado de Alcance Regional. Creado por el Tratado de Montevideo como sustitución de la Asociación Latinoamericana de Libre Comercio (ALALC).

Para ayudar con la consolidación de un Mercado Común Latinoamericano, ALADI contempló tres mecanismos de integración a nivel económico que a continuación se enumeran:

Preferencia arancelaria regional (PAR)-ALADI: Son los que aplican disminución de gravámenes arancelarios para las importaciones de terceros países (Asociación Latinoamericana de Integración, 2015).

Acuerdos de alcance regional (AAR)- ALADI: Son los suscritos entre los países miembros de la Asociación Latinoamericana de Integración (ALADI). Se 
han firmado: Las Nóminas de Apertura de Mercados, Preferencias de Alcance Regional, Acuerdo Regional de Cooperación Científica y Tecnológica, Acuerdo Regional de Cooperación e Intercambio de Bienes, en las Áreas Cultural, Educacional y Científica y Acuerdo Marco para la Promoción del Comercio (Asociación Latinoamericana de Integración, 2015).

Acuerdos de Alcance Parcial (AAP)- ALADI: Son en los que participan dos o más países miembros, y definen un tratamiento preferencial a las importaciones de productos originarios de algunas de las partes del Acuerdo, con el fin de promover el comercio, el desarrollo económico y productivo de los países. Propenden a crear las condiciones necesarias para profundizar el proceso de integración regional. Estos Acuerdos de Alcance Parcial abarcan tanto desgravación arancelaria como promoción del comercio y el turismo; intercambio agropecuario; complementación económica; cooperación aduanera, tributaria, tecnológica, financiera, científica y sanitaria; normas; técnicas y régimen de origen (Asociación Latinoamericana de Integración, 2014).

De otro lado, el 26 de marzo de 1991, se conformó el Mercado Común del Sur (Mercosur), proceso de integración regional creado, inicialmente por Brasil, Argentina, Paraguay y Uruguay, posteriormente se incorporó Venezuela y recientemente Bolivia está en proceso de adhesión (Declaración 2308, Mercado Común del Sur, 2000). Sus objetivos se concentran en la libre circulación de bienes y servicios por medio de la eliminación de las barreras comerciales, la creación de un arancel externo común (AEC), sistema por el cual se clasifican e identifican todo el universo de mercancías. Las mercancías identificadas tienen adjudicada un alícuota valor, expresado en porcentaje, llamado arancel que es el impuesto que paga cada mercancía al ingresar al Mercosur por cualquiera de sus Estados Partes. Los aranceles actualmente siguen ubicándose entre el 0\% de piso fiscal y el 20\% de techo arancelario desde su reglamentación en el año de 1998 (Uruguay, 1998).

El 1 de enero de 1995, surge el Grupo de los tres (G-3) (Comunidad Andina de Naciones, 2015), como un acuerdo de libre comercio que tiene como primer momento a Colombia, México y Venezuela. Acuerdan una reducción de nuevos aranceles durante diez años (a partir de 1995) para el comercio de bienes y servicios entre sus miembros. El acuerdo de tercera generación no se limita a la liberalización del comercio, sino que incluye temas como inversiones, servicios, compras gubernamentales, normas para luchar contra la competencia desleal y los derechos de propiedad intelectual.

El presidente de Venezuela aprovechó una reunión con sus colegas de Bolivia, Paraguay y Uruguay, celebrada el 19 de abril de 2006, para, de forma 
sorpresiva, anunciar su salida de la Comunidad Andina de Naciones (CAN). Diez días después, la medida fue seguida del anuncio del presidente boliviano Evo Morales sobre la nacionalización de los hidrocarburos. Venezuela insiste en que su salida de la CAN es consecuencia directa de los perjuicios inmediatos que le causan los tratados de libre comercio (TLC) firmados por Colombia y Perú. (Malamud, Oscar, 2015).

Por su parte, basada en la historia compartida y solidaria de las naciones latinoamericanas, contando con valores multiétnicos, multilingüe y multiculturales, que luchan por la emancipación y la unidad de América del Sur, en honor a la visión de quienes forjaron la independencia y libertad a favor de esa unión y la construcción de un futuro común, e inspirada por la Declaración de Cuzco (8 de diciembre, 2004), Brasilia (30 de septiembre de 2005) y Cochabamba (9 de diciembre de 2006), se establece la Unión Sudamericana de Naciones, con fecha oficial del 11 de marzo de 2011. Esta tiene como objetivo construir, de forma participativa y consensuada, un espacio de integración y unión en el diálogo político cultural, social, económico y político entre sus pueblos, dando prioridad a las políticas sociales, la educación, la energía, la infraestructura, el financiamiento y el medio ambiente, entre otros (UNASUR, 2015).

De otro lado, nace la Comunidad de Estados Latinoamericanos y Caribeños, conocida por sus siglas CELAC, organización regional intergubernamental que reúne a los países de América Latina y el Caribe, sin la injerencia de Estados Unidos y Canadá. De esta forma, se establece un foro que agrupa a los 33 Estados soberanos de la región, y que busca profundizar la integración política, económica, social y cultural de América Latina y el Caribe (Cancillería, 2015).

Un grupo que ha venido tomando fuerza en la región ha sido la Alianza del Pacifico que, por iniciativa del presidente de Perú, Alan García, invitó a Chile, Colombia y México a participar en un esquema de integración que promueva las relaciones económicas con los países en el "otro lado" del Pacífico, y aspira ser la octava economía del mundo (Ministerio de Industria, 2015). El pasado 20 de junio, la Organización para la Cooperación y el Desarrollo Económicos (OCDE) y los cuatro países que conforman la Alianza del Pacífico firmaron un convenio en materia de apoyo de las micro, pequeñas y medianas empresas (Mipymes). El propósito del acuerdo es contar con mayor integridad y competitividad, toda vez que ese sector es un importante motor para la creación del empleo y coadyuvante en el crecimiento y el desarrollo económico de los países donde se sitúan (NOTIMEX, 2015).

Por otra parte, hoy Costa Rica y Panamá ostentan la posición de observadores-candidatos. Guatemala ha anunciado su interés de ser miembro a Chile 
y a México. En la región de las Américas, los únicos países que cumplen plenamente con el único requisito de adhesión formal son Canadá y Estados Unidos. Por primera vez Canadá tiene una oportunidad histórica de integrarse, consolidarse y visibilizarse en Latinoamérica, si pide admisión plena en la Alianza Pacífico (Portafolio, 2015).

\section{Alianza o no Alianza: ¿Esa es la cuestión?}

Hasta qué punto la Alianza del Pacífico logra su objetivo general para la libre circulación de bienes, servicios, capitales y personas. Esta reciente iniciativa para la integración económica y la cooperación en América Latina y su enfoque de libre mercado a la integración regional ha llegado con frecuencia a ser vista como un contrapeso o, al menos, una alternativa al estancamiento ocurrido con el MERCOSUR; restringiendo la firma de acuerdos bilaterales de sus socios con terceros mercados, es el caso con la Unión Europea.

En este contexto, se han impedido la firma de un posible tratado entre ambos bloques. Así mismo, se observa la Alianza del Pacifico como la competencia al liderazgo de Brasil en la región y como una barrera a la consolidación de la Unión de Naciones Sudamericanas (UNASUR) y a la Comunidad de Estados Latinoamericanos y del Caribe (CELAC).

Para el catedrático Ramón Tamames, la integración económica es una de las alternativas en la alianza de la región a nivel comercial entre los Estados, de manera conjunta con el librecambio y la cooperación económica. "'La integración económica' consiste específicamente en un proceso en el que dos o más mercados nacionales previamente separados y de dimensiones unitarias poco adecuadas se unen para formar un solo mercado (mercado común) de una dimensión más idónea“ (Tamames, 2000, p. 48).

El peso y la dinámica de las transacciones comerciales internacionales aún no se reflejan plenamente en las normas del sistema multilateral de comercio. Los acuerdos siguen operativos, basados principalmente en una lógica tradicional: la predicción de reconocimiento bilateral de origen. Los modestos resultados alcanzados en la Ronda Uruguay en disciplinas tales como servicios, inversiones y propiedad intelectual y la retirada de los Estados Unidos de la Ronda de Doha en 2008 muestran la dificultad de multilateralizar estos temas.

Desde el impasse generado por la paralización de Doha, las principales economías del planeta, centros de operaciones en las estructuras (en particular los Estados Unidos) han tenido que acelerar la firma de los acuerdos regionales, 
la promoción de la gobernanza global ad hoc. Acuerdos regionales recientes firmados por los EE.UU., la UE, China y la India han profundizando la regulación en zonas que ya son parte de la agenda de la Organización Mundial del Comercio (OMC), tales como las normas de origen, salvaguardias, servicios, propiedad intelectual (lo que es -denominado "OMC-plus)"; y la ampliación de la frontera regulatoria en nuevos temas, por ejemplo, la competencia, la contratación pública, el medio ambiente y las normas laborales, lo que ha sido llamado “extra-OMC" por el catedrático Manuel Sánchez. Los Estados Unidos y la UE, por ejemplo, han dirigido una atención especial a los nuevos temas y, en profundidad, a los compromisos en materia de propiedad intelectual. A su vez, China e India han evitado entrar en nuevos temas, prefiriendo centrarse en la regulación de las subvenciones (Sánchez, 2012).

El valor acumulado de dichos acuerdos -llamada "integración profunda" o "acuerdos comerciales del siglo XXI" (Baldwin, 2011) - ya no se centra en las preferencias arancelarias, pero sí en la armonización de las políticas, normas y estándares nacionales básicas de fluidez. En este sentido, los requisitos de estos acuerdos no pueden coincidir con el nivel de crecimiento institucional o de la estrategia de los países en desarrollo, ya que pueden imponer limitaciones a la autonomía de estos países en la gestión de las políticas públicas.

En Latinoamérica, los presidentes Evo Morales y Rafael Correa han criticado la Alianza Pacífico, al considerar que la reciente entrada de los Estados Unidos en las negociaciones del Acuerdo de Asociación Transpacífico ([TPP, por sus siglas en Inglés], que también tiene 11 miembros del Foro Asia-Pacífico de Cooperación Económica [APEC, siglas en Inglés]), parecen indicar el inicio de una segunda fase del regionalismo, caracterizada por la integración a través de estrategias plurilaterales, entre los más importantes centros de comercio internacional y que, precisamente, por medio de El Acuerdo Transpacífico de Cooperación Económica (también conocido comoTPPpor sus siglas en inglés), la inserción de Estados Unidos, podrá fortalecerse más en la región (Arce, 2013).

Como cualquier iniciativa de integración, la Alianza del Pacífico cuenta con conceptos de respaldo y en contra. El Parlamento Europeo, por ejemplo, señala, en una sesión informativa, que la dominación comercial de México podría ser una preocupación para la Alianza: en 2012, las exportaciones mexicanas representaron el 67\% de todas las exportaciones de la Alianza del Pacífico, y sus exportaciones a los EE.UU. por sí solo cuentan por más de $50 \%$ del valor total de las exportaciones del bloque comercial (CANCILLERIA, 2015). Esto podría plantear problemas de Colombia, Chile y Perú, en la medida en que abran sus fronteras a la influencia externa de una economía dominante, al igual que México, dentro de la Alianza. Este es solo uno de muchos acuerdos comerciales 
que Colombia ha suscrito en los últimos años; acuerdos comerciales con los EE.UU. también se han plagado de críticas similares.

"El continente se ha dividido", señala el expresidente brasileño Henrique Cardoso en referencia al nacimiento de la Alianza del Pacífico (Valor, 2012). El presidente peruano Ollanta Humala se reunió con Luiz Inácio Lula da Silva, en el marco del foro "Diez años de la alianza estratégica entre Brasil y Perú, 2003-2013" recordó que en diez años "tenemos gran progreso logrado en la integración peruana-brasileña y sobre todo en el entendido de que es una alianza natural para la creación de un bloque de dos océanos Atlántico y el Pacífico" (La Voz de Rusia, 6 de Junio de 2013).

Los datos sobre la inversión extranjera directa (IED) se pueden considerar una radiografía de la región. La distribución física internacional ha aumentado exponencialmente en América del Sur, pasando de un poco más de 30 mil millones de dólares en los primeros años de la década de 2000, hasta 143 mil millones en el 2012. Esto significa que se ha quintuplicado, según el último informe de la CEPAL (Industriales, 2014).

Los tres países andinos de la Alianza del Pacífico pasaron de una inversión extranjera directa de 11 mil millones de dólares a principios de este siglo, a un poco más de 58 mil millones de dólares. Este es el mayor crecimiento en la región. Pero lo que revela el carácter de las economías nacionales es el sector al que esta inversión se dirige.

De igual manera, aún quedan importantes dudas sobre el nivel de integración profunda que se ha anunciado, porque hasta el momento solamente se ha desarrollado una zona de libre comercio (Pastrana \& Gehring, 2014); sin embargo, los presidentes de los 4 países miembros son conscientes de esta situación, ya que, en la declaración final de la X Cumbre, establecieron su deseo de seguir trabajando por perfeccionar la iniciativa de integración profunda, tendiente a mejorar los aspectos de cooperación, capital humano y seguridad; entre otros temas (Alianza, 2015).

\section{Relaciones América Asia-Pacífico}

El creciente protagonismo de los países en desarrollo en la economía mundial no solo ha elevado su dinamismo económico, sino también vínculos más fuertes a través de una mayor cooperación Sur-Sur, el comercio, la inversión y la cooperación. Los países en desarrollo, tanto en América Latina y Asia del Este, deben actuar de forma proactiva para prepararse para este nuevo escenario, 
ajustando sus políticas y estrategias con el fin de aprovechar el potencial de crecimiento de los vínculos Sur-Sur. En este sentido, las autoridades de ambas regiones deben redoblar sus esfuerzos para identificar y capitalizar sobre sus potenciales complementariedades, mediante la creación de alianzas comerciales birregionales, mejorando la cooperación en innovación y capital humano, la calidad de flujos comerciales y de inversión, y ayudando a crear condiciones más estables para el crecimiento.

En este sentido, el Foro de Cooperación América Latina-Asia del Este (FOCALAE) puede y debe desempeñar un papel de liderazgo como centro de coordinación para la estructuración de una agenda de cooperación birregional.

Los países de América Latina pasaron de una economía cerrada a una abierta sin transiciones, mientras que Asia lo hizo de manera paulatina. Solo recientemente, en 1997, cuando la crisis asiática, buscaron la ayuda del FMI para respaldar las monedas que sufrían una caída en su cotización y se pudieron implementar algunas medidas del "Consenso de Washington". Ambos continentes consideran la adquisición de tecnología como un vehículo para el desarro1lo. Esto en Asia significa un control y selectividad en la elección de empresas transnacionales, de modo que cumplan con la transferencia de tecnología. En cambio, en América Latina se apuesta más para que su desarrollo se encuentre hacia el producto y las exportaciones, pero se puso poco énfasis en el desarrollo de capacidades de absorción tecnológica (Guillén, 2008).

Con respecto a los países de la Alianza Pacífico, cada uno de los miembros cuenta con una relación diferenciada con Asia Pacífico. Empezando por Chile, a nivel histórico, en las relaciones entre Chile y Asia se puede apreciar que en el curso del siglo XIX se dio cierto desarrollo de la navegación entre puertos chilenos y del Oriente, pasando por diversos territorios insulares y tocando ocasionalmente en la colonia de Australia (Nueva Gales del Sur). Sin embargo, los flujos comerciales originados en estos viajes fueron esporádicos y de escaso volumen, y las corrientes de circulación de personas no llegaron a ser importantes (Wilhemy, 2010).

En el último cuarto del siglo XX Chile se considera como un país pionero en las relaciones de Asia-Pacífico, y comenzó a construir la idea de la política exterior chilena como una política "diversificada" y "exitosa", caracterizada por un interés permanente en ampliar y diversificar mercados internacionales a través de una rápida y profunda liberalización comercial. Esta constante de la política exterior y comercial chilena conduce a que el país no quiera ser miembro pleno de ningún proyecto regional que implicase la coordinación de políticas comerciales, con excepción de su aspiración de pertenecer al NAFTA. 
Como consecuencia, Chile fue el país latinoamericano que más temprano firmó acuerdos comerciales con los demás países de la región, y también el que ha desarrollado más temprana y profundamente sus relaciones comerciales con Asia-Pacífico (Prieto \& LADINO, 2014).

En el caso de México con Asia, al comienzo de su historia, las relaciones internacionales de México referidas a Asia han sido escasas, salvo algunas excepciones, como sucedió con las migraciones de origen asiático durante las primeras décadas del siglo XX. En el marco de relaciones internacionales, las alianzas intergubernamentales han sido el elemento dominante. Después del 11 de septiembre de 2001, la agenda del Foro de Cooperación Económica Asia Pacífico, conocido por sus siglas en inglés, como APEC, ha estado relacionada con el campo de la liberalización comercial, financiera y al de la cooperación política en una amplísima gama de temas relacionados con la seguridad tradicional y no tradicional; los asuntos tratados van desde la prevención del terrorismo internacional hasta las pandemias (sida, gripe aviar), pasando por la represión del crimen organizado, del narcotráfico, del tráfico de personas o de la corrupción.

Por otra parte, para Perú, la relación con Asia lleva bastantes décadas de desarrollo; a nivel histórico, fue el primer país latinoamericano que aceptó inmigración masiva desde China, y de 1849 a 1872 se calcula llegaron al Perú unos 100,000 ciudadanos de ese país, como trabajadores (culíes) y, por otro lado, desde 1899 empezaron a arribar ciudadanos japoneses, y se calcula que hasta la segunda década del siglo XX llegaron en un numero de 25.000. Esto ha hecho que el Perú tenga la comunidad china más grande de Latinoamérica, y la segunda más grande de nikkei (descendientes de japoneses), después de Brasil, en la región. De 1990 en adelante empezaron a llegar también coreanos, aunque su número es pequeño aún (Aquino, 2012). El comercio con Asia creció de forma extraordinaria. Esto ha sido resultado de las políticas de apertura comercial que se implementaron desde 1990 en adelante, resultantes en tratados de libre comercio (TLC), que el país ha firmado, un total de 54 a fines del 2012, y de la gran demanda por las materias primas que el Perú posee (Aquino, 2012).

En el caso de Colombia, la política exterior con la región transpacífica ha emprendido varias estrategias de inserción internacional en Asia Pacífico con más énfasis en la búsqueda de la admisión al Foro de Cooperación Económica Asia Pacífico (APEC, por sus siglas en inglés). En el siglo XXI, los sistemas de cooperación internacionales y regionales siguen generando grandes cambios, razón por la que el país debe ser coherente con estos procesos. Para ello, los actores y los interesados tienen que realizar un diagnóstico completo, con el fin de formular una estrategia prospectiva para diversificar la agenda de las relaciones con esta región del planeta. La llamada es ir más allá del significado tradicional 
dado a APEC, a fin de garantizar una mejor lectura de las próximas etapas de la cooperación a través del Pacífico (Pastrana \& GEHRING, 2014).

Las relaciones entre Colombia y Asia-Pacífico se inician en 1908 con el tratado de amistad, comercio y navegación suscrito con Japón. Ya hacia finales del siglo XX, el plan de desarrollo propuesto por el presidente Ernesto Samper propone avanzar en el establecimiento de mayores vínculos de cooperación con los países de las cuencas del pacífico, Colombia recibe la presidencia del movimiento de los no alineados. Posteriormente, Andrés Pastrana menciona que el gobierno colombiano aprovechará la reestructuración que la crisis asiática impone sobre las economías del Pacífico. Finalmente, el presidente Álvaro Uribe propone consolidar la inserción colombiana en el Pacífico, promover intereses comerciales a través de las embajadas regionales y el ingreso de Colombia a la cooperación económica del Asia Pacífico (Pastrana, Colombia, 2012).

Sin embargo, uno de los grandes retos de Colombia para acercarse al continente asiático y depender cada vez menos de Estados Unidos es que el país realice un reajuste en el objetivo y despliegue una estrategia que permita alcanzarlo; ello resulta particularmente difícil para un país como Colombia, dado que muchos de los Estados que están contribuyendo a hacer más multipolar el mundo están ubicados en Asia-Pacífico. Uno de los pasos estratégicos para alcanzar el gran objetivo es superar el rezago de solo mirar en política exterior al país del norte, lo que ha producido el relacionamiento con esta cuenca (Barbosa, 2011).

\section{Conclusiones y Recomendaciones}

Con el fin de fomentar el crecimiento y promover las exportaciones en un mundo globalizado, los acuerdos comerciales se están convirtiendo cada vez en más populares para las economías. El bloque comercial de la Alianza del Pacífico se convierte en un órgano principal de comercio para el trato con la región latinoamericana. El potencial es enorme, ya que los cuatro países fundadores de la Alianza del Pacífico -Chile, Colombia, México y Perú- representan casi el 36 por ciento del PIB de América Latina. Sin embargo, otros líderes de los países del continente como Evo Morales y Rafael Correa han expresado su preocupación por su apreciación de que el nuevo bloque comercial no es tanto una iniciativa económica, sino una herramienta política.

La Alianza del Pacífico está buscando impulsar el comercio en varias economías latinoamericanas. Pero algunos líderes latinoamericanos están preocupados. La razón es, simplemente, que su diseño causa división geopolítica entre los gobiernos autoritarios y gobiernos de derecha. En particular, dijo el presidente de 
Bolivia, Evo Morales en el Foro de São Paulo, en 2013, que la Alianza del Pacífico era un "esquema geopolítico" a través del cual los EE.UU. podrían oponerse a los gobiernos de izquierda de Brasil, Argentina, Uruguay, Bolivia, Venezuela y Ecuador.

Esto, en marcado contraste con las economías regionales como Brasil y Argentina, que están empleando las políticas orientadas hacia el interior, así como las posturas proteccionistas que reducen el comercio transfronterizo, que han deteriorado su crecimiento económico. Es interesante, dado que ambos países son miembros clave del bloque Mercado Común del Sur (MERCOSUR).

Como se ha mencionado, varios jefes de Estado han criticado la Alianza por ser una herramienta política, y no solo un órgano para promover la cooperación económica. El Foro de Sao Paulo, que es una conferencia de los partidos políticos de izquierda y organizaciones de América Latina y el Caribe, fue escenario de un acalorado debate de los objetivos y del impacto de la Alianza del Pacífico, del cual los líderes estatales manifestaron que era un intento de recrear el Consenso de Washington en Suramérica.

En conclusión, es importante destacar que los proyectos de integración no pueden darse sin una visión política de convertirse en un agente en relaciones internacionales. América Latina tendría que tener una voz muy clara de región en el orden mundial, que tiende a una regionalización, unida a la existencia de un liderazgo latinoamericano. Colombia está en una posición central de América Latina, cuenta con la tercera población más importante de la región. Hoy, ya no existe un enfoque hegemónico; en la lucha entre el Pacífico y el Atlántico, aunque si bien sigue vigente, se reconoce que el Pacífico ha ido ganando la pugna con el redireccionamiento estratégico de las potencias de Asia-Pacífico. En este contexto, si se permanece ofreciendo solamente commodities a esta región, dejará de generar importancia y no continuará hacia lo que debería ser su principal objetivo: empoderar a la región y hacer que América Latina, cada vez más, logre un mayor peso en los escenarios multilaterales.

Precisamente, uno de los mayores problemas que tiene Colombia, frente a los demás miembros de la Alianza Pacífico, es, según Michael Fairbanks, consejero en competitividad del gobierno del presidente César Gaviria, la existencia de una amplia necesidad en el país de "creer en la competitividad como fuerza de cambio positivo, fomentar la iniciativa y la innovación" (Fairbanks, Michael, Julio 20; Portafolio, 2015, p. 13).

No obstante, en todos los países de América Latina se pueden establecer grandes desafíos debido a la contracción económica de la región, ya que en el 2015, la 
región crecerá solamente un $0,5 \%$. Entre los países, Panamá liderará la expansión regional con un alza de 6,0\%, seguido de Antigua y Barbuda (5,4\%), República Dominicana y Nicaragua (ambos con 4,8\%). México alcanzará un producto interno bruto (PIB) de $2,4 \%$ y Argentina de $0,7 \%$. Brasil anotará una contracción de $-1,5 \%$, mientras que Venezuela tendrá una disminución aún mayor de $-5,5 \%$ (CEPAL, 2015). Por lo tanto, dependerá de las estrategias de los países de la Alianza Pacífico fortalecer, en el tiempo, su modelo de integración o permitir que este se debilite, hasta pasar por una tendencia temporal que se difumine en el tiempo. 


\section{Referencias}

Alianzadelpacìfico.net. (12 de 07 de 2015). Recuperado de http://alianzapacifico. net/presidentes-de-la-alianza-del-pacifico-suscriben-la-declaracion-de-paracas-en-el-marco-de-la-x-cumbre-de-este-mecanismo/

Arce, L. (2013). Hacia una mayor integración regional? Las implicancias de un acuerdo de inversiones y comercio entre EEUU y UE. Asunción, Paraguay: Observatorio de Economía Internacional - Centro de Análisis y Difusión de la Economía Paraguaya.

Baldwin, R. (2011). Regionalismo siglo 21: Llenar la brecha entre el comercio del siglo 21 y las reglas comerciales del siglo 20. OMC.

Barbosa, F. (2011). La inserción de Colombia en el Asia Pacífico 2020: Colombia en el nuevo océano. En Fernando Barbosa (Ed.), La inserción de Colombia en el Asía Pacífico 2020: Colombia en el nuevo océano (pp. 21-31). Bogotá, Colombia: Universidad Jorge Tadeo Lozano.

Aquino, C. (21 de 04 de 2012). Universidad Nacional Mayor de San Marcos. Recuperado de http://economia.unmsm.edu.pe/

Cancillería. (22 de 02 de 2015). Recuperado el 10 de 05 de 2015, de http://www. cancilleria.gov.co/international/consensus/clacs

CANCILLERIA. (29 de 06 de 2015). Recuperado de http://www.cancilleria.gov.co/ international/consensus/pacific-alliance

CEPAL, E. M. (2015). El Mercado Común del Sur, situación actual y perspectivas, Sector de Asesoría Técnica. Buenos Aires: CEPAL.

CEPAL. (22 de 10 de 2015). CEPAL. Recuperado de http://www.cepal.org/es/comunicados/america-latina-y-el-caribe-crecera-solo-05-en-2015

Guillén, H. (2008). Políticas y estrategias de desarrollo en América Latina y Asia. Realidad Económica (237).

Industriales, Cámara de (21 de 05 de 2014). Confederación de Cámaras Industriales. Recuperado de http://www.concamin.mx/sintesis.php

Industriales, Cámara de (21 de 05 de 2014). Confederación de Cámaras Industriales. Recuperado de http://www.concamin.mx/sintesis.php

Ministerio de Industria, Turismo y Comercio (08 de 03 de 2015). MINCIT. Recuperado de 2015, de MINCIT: http://www.mincit.gov.co/tlc/publicaciones. php?id=7180\&dPrint $=1$ 
NOTIMEX. (20 de 06 de 2015). NOTIMEX. Recuperado de http://www.notimex. com. $\mathrm{mx} / \mathrm{acciones} / \mathrm{verNota.php?clv=136611}$

Pastrana, E. (2012). Colombia: ¿Una potencia en desarrollo? Escenarios y desafios para su política exterior. Fundación Konrad Adenauer.

Pastrana, E., \& GEHRING, H. (2014). Alianza del Pacifico: Mitos y realidades. Cali, Colombia: Universidad Santiago de CalI.

Portafolio. (19 de 05 de 2015). Portafolio. Recuperado de http://www.portafolio.co/ opinion/canada-nuevo-miembro-la-alianza-del-pacifico

Prieto, G., \& LADINO, N. (2014). La proyección de Chile en Asia-Pacífico. En E. PASTRANA, \& G. Hubert, Alianza del Pacifico: mitos y realidades. Cali, Colombia: Editorial Universidad Santiago de Cal.

Tamames, R. (2000). Estructura económica internacional. Madrid, España: Alianza Editorial.

Tirado, A. (1997). Integración y democracia en América Latina y el Caribe. Buenos Aires, Argentina: INTAL.

Sánchez, M. (2012). Compromisos de las economías grandes y medianas empresas en los acuerdos comerciales preferenciales: El contraste entre la Unión Europea y los Estados Unidos, China y la India. En M. SANCHEZ, Documento de trabajo $N^{o} 1700$. Buenso Aires, Argentina: IPEA.

UNASUR. (22 de 01 de 2015). UNASUR. Recuperado de http://www.unasursg.org/

Uruguay, R. A. (21 de 02 de 1998). Real Academia Uruguaya. Recuperado de http:// www.rau.edu.uy/mercosur/faq/pre16.merco.htm

VAN MARREWIJK, C. (2009). The Princeton Encyclopedia of the World Econo$m y$ (Vol. 2). United States of America: Princeton.

Wilhemy, M. (2010). La trayectoria de Chile frente a la región Asia-Pacífico. Estudios Internacionales, 167, 125-167. 